\begin{tabular}{|c|c|}
\hline Title & $\begin{array}{l}\text { Material Gain A naly sis of GeSn/SiGeSn Quantum W ells for Mid-Infrared Si-Based Light Sources Based on Many- } \\
\text { Body Theory }\end{array}$ \\
\hline Author(s) & Fujisawa, T akeshi; Saitoh, Kunimasa \\
\hline Citation & $\begin{array}{l}\text { IEEE journal of quantum electronics, 51(5), 1-8 } \\
\text { https://doi.org/10.1109/JQE.2015.2410283 }\end{array}$ \\
\hline Issue Date & 2015-05 \\
\hline Doc URL & http:/hdl.handle.net/2115/58902 \\
\hline Rights & $\begin{array}{l}\text { (c) } 2015 \text { IEEE. Personal use of this material is permitted. Permission from IEEE must be obtained for all other uses, in } \\
\text { any current or future media, including reprinting/republishing this material for advertising or promotional purposes, } \\
\text { creating new collective works, for resale or redistribution to servers or lists, or reuse of any copyrighted component of } \\
\text { this work in other works. }\end{array}$ \\
\hline Tyре & article (author version) \\
\hline File Information & fujisawa final.pdf \\
\hline
\end{tabular}

Instructions for use 


\title{
Material Gain Analysis of GeSn/SiGeSn Quantum Wells for Mid-Infrared Si-Based Light Sources Based on Many-Body Theory
}

\author{
T. Fujisawa, Member IEEE, and K. Saitoh, Member IEEE
}

\begin{abstract}
Material gain of GeSn/SiGeSn quantum wells, which can be grown on Si substrate by using a buffer layer, is analyzed based on microscopic many-body theory (MBT) for mid-infrared light sources based on Si photonics. MBT can take into account a gain spectrum broadening associated with scattering phenomena, such as Coulomb scattering, based on quantum field theory, and does not need any artificial fitting parameters, such as a relaxation time, used in conventional analysis. Not only $\Gamma$ - but also carrier distributions in L-points are considered for the gain analysis. By using MBT, the quantum well structures maximizing the material gain and the differential gain at the threshold are investigated in terms of the well thickness, the strain, and the energy difference of quantum states between $\Gamma$ - and $L$-points.
\end{abstract}

Index Terms - Mid-infrared photonics, Si photonics, GeSn quantum wells, and many-body theory.

\section{INTRODUCTION}

$\mathrm{M}$ ID-infrared (Mid-IR) photonics using 2 to $5 \mu \mathrm{m}$ wavelength region is an emerging research area and have attracted great attention for an environmental gas sensing and new wavelength optical communication system using photonic bandgap fiber [1]. Optical devices for mid-IR region have also been developed by using conventional III-V material system [2] and Si [3]. Especially, devices using Si-photonics technology have been intensively studied since Si waveguides do not suffer from two-photon absorption in this wavelength range and they can be fabricated in a CMOS environment, leading to lower cost of the devices. In [3], some passive components using $\mathrm{Si}$ waveguides such as arrayed waveguide grating and concave grating, GeSn photo-diodes, and hybridly integrated GaSb light sources fabricated on Si substrate were demonstrated.

Among them, light sources are crucial components for both sensing and communication applications. Although the hybrid integration of other material light sources on $\mathrm{Si}$ is very promising, a monolithic integration of light sources is also an attractive candidate due to its integrability with other components. As a one candidate for monolithically integrated light sources on $\mathrm{Si}$, recently, tensile-strained and n-doped $\mathrm{Ge}$ laser grown on Ge-buffer on Si substrate was reported [4]. Ge, inherently indirect bandgap material, is transformed to

Manuscript received October 9, 2015. Authors are with the Graduate school of information science ant technologies, Hokkaido University, North 14 West 9, Sapporo, Hokkaido, 060-0814 Japan (e-mail: fujisawa@ist.hokudai.ac.jp). quasi-direct bandgap material by following ways. The band edge energy difference between $\Gamma$ and $L$ points are decreased by introducing tensile strain into Ge active layer. Also, the Ge is heavily n-doped $\left(4 \times 10^{19} \mathrm{~cm}^{-3}\right)$ to fill the bandedge of $\mathrm{L}$ band with carriers. As a consequence, Ge becomes quasi-direct bandgap material and the lasing was realized around $1.7 \mu \mathrm{m}$.

By introducing small amount of Sn into Ge, it is known that the bandgap (of GeSn) is drastically reduced and reaches mid-IR region [5]. Also, GeSn with Sn fraction exceeds about $10 \%$, the $\Gamma$-bandgap becomes smaller than that of L-bandgap, and $\mathrm{GeSn}$ becomes direct bandgap material without any strain and doping. Direct transition photoluminescence and p-doping were experimentally observed and realized on GeSn buffer layer grown on Si substrate [6]. Therefore, GeSn is a promising material for Si-based mid-IR light sources.

To extract the ultimate performance of this material system as light sources, the optimum design of quantum wells (QWs) to maximize the optical gain is necessary. Also, the optimization of differential gain is important for high-speed applications since the modulation speed of the laser is directly influenced by the differential gain [7]. Although the preliminary studies investigating material gain of GeSn QWs have been reported $[8,9]$, the optimization of quantum structures to maximize the material gain and differential gain has not been done.

Furthermore, in these studies, so-called free-carrier theory (FCT) based on Fermi's golden rule was used to obtain material gain of QWs. Although FCT is easy to use for obtaining optical properties of QWs, it needs an experimental fitting parameter (relaxation time) associated with optical spectrum broadening originating from scattering phenomena in semiconductors. Results obtained by FCT are greatly affected by the fitting parameter (shown later), and it was demonstrated that spectral positions and the spectral shapes obtained by FCT are not consistent with the experiment [10-14]. Therefore, it is difficult to grasp the inherent optical properties of GeSn QWs if FCT is used for the analysis.

In this paper, the material gain of $\mathrm{GeSn} / \mathrm{SiGeSn}$ QWs is investigated for mid-IR Si-photonics light sources by using microscopic many-body theory (MBT) [10-15]. MBT does not need any fitting parameters used in FCT to obtain optical properties of QWs and only needs basic bulk band parameters. It has been shown that MBT can explain experimental results very well both qualitatively and quantitatively without fitting parameters for QWs made with conventional III-V material system $[10,11]$. Here, to treat group IV material, the carrier 
distribution in L-band is taken into account in the MBT. By using MBT, the QW structures maximizing the material gain and the differential gain are investigated in terms of well thickness, the strain, and the energy difference of quantum states between $\Gamma$ - and L-bands.

\section{ANALYSIS METHODS}

\section{A. Band structure calculation}

Figure 1 shows the layer structure considered in this paper. GeSn buffer layer is placed on $\mathrm{Si}$ substrate and $\mathrm{GeSn} / \mathrm{SiGeSn}$ QWs are grown on the buffer layer. The barrier and separate confinement heterostructure (SCH) layers are assumed to be lattice matched to the buffer layer. The bandgap wavelength of the barrier and SCH layers are 2.3 and $2.2 \mu \mathrm{m}$. The compositions of barrier and $\mathrm{SCH}$ are $\mathrm{Si}_{0.1} \mathrm{Ge}_{0.76} \mathrm{Sn}_{0.14}$ and $\mathrm{Si}_{0.14} \mathrm{Ge}_{0.7} \mathrm{Sn}_{0.16}$. The basic band parameters of Ge, $\mathrm{Si}$, and $\mathrm{Sn}$ are taken from [9] and [16]. Figure 2 (a) shows the bulk bandgap energy of GeSn as a function of Ge mole fraction for $\Gamma$ and $\mathrm{L}$ points. Both bandgaps are reduced for smaller Ge mole fraction. At the Ge composition of 0.88 , the bandgap energy of $\Gamma$ and $L$ points becomes the same and bulk GeSn becomes direct gap material for the Ge composition smaller than 0.88 . Therefore, for the buffer layer, $\mathrm{Ge}_{0.88} \mathrm{Sn}_{0.12}$ is chosen in this paper. Figure 2 (b) shows the bandgap energy of bulk GeSn on $\mathrm{Ge}_{0.88} \mathrm{Sn}_{0.12}$ buffer as a function of Ge composition. The energy change due to strain is taken into account. For Ge composition smaller than 0.88 (compressive strain), $\Gamma$ bandgap is smaller than that of $\mathrm{L}$, therefore, GeSn is still direct gap material even if the strain effect is considered.

The band structure of $\Gamma$-band is calculated by $\boldsymbol{k} \cdot \boldsymbol{p}$ theory $[9,13,16]$ with the axial approximation. For Ge-based QWs, since the bandedge energy of L-band is very close to that of $\Gamma$-band, the carrier distribution in L-band has to be taken into account $[8,9]$.We solve following effective mass equation to obtain the conduction band structure of L-band:

$$
\begin{aligned}
& H_{L} \phi_{L}=E_{L} \phi_{L} \\
& H_{L}=-\frac{\hbar^{2} k_{z}^{2}}{2}\left(\frac{1}{3 m_{L L}}+\frac{2}{3 m_{t L}}\right)+j \frac{\sqrt{2} \hbar^{2} k_{1} k_{z}}{6}\left(\frac{1}{m_{L L}}-\frac{1}{m_{L L}}\right) \\
& +\frac{\hbar^{2} k_{1}^{2}}{2}\left(\frac{2}{3 m_{L L}}+\frac{1}{3 m_{L L}}\right)+\frac{\hbar^{2} k_{2}^{2}}{2 m_{t L}}+V_{L}(z)
\end{aligned}
$$

where $\phi_{L}$ and $E_{L}$ are the wavefunction and the subband energy of L-band, $k_{z}$ is the wavenumber for growth direction, $k_{1}$ and $k_{2}$ are transverse wavenumber, $m_{L L}$ and $m_{t L}$ are longitudinal and transverse effective masses at L-bandedge, and $V_{L}$ is the bandedge potential distribution including strain effects. We used the model solid theory [17] to obtain bandlineups of $\Gamma$ - and L-bands.

Electron density, $N_{e}$, in the conduction band is given by

$$
N_{e}=\sum_{n} \int \frac{k_{t}}{\pi L_{w}} f_{e}\left(E_{\Gamma, n}\right) d k_{t}+\sum_{m} \int \frac{8}{(2 \pi)^{2} L_{w}} f_{e}\left(E_{L, m}\right) d k_{1} d k_{2}(2)
$$

where $k_{\mathrm{t}}$ is the transverse wavenumber for $\Gamma$-band, $L_{w}$ is the well thickness, $f_{e}(E)$ is the Fermi distribution for electrons, $n$ and $m$ are the QW subband number of $\Gamma$ - and L-bands. First term and second term of right hand side of (2) correspond to the carrier density in $\Gamma$ - and L-bands. The hole density, $N_{h}$, in the valence

\begin{tabular}{|c|}
\hline SiGeSn SCH \\
\hline SiGeSn Barrier \\
\hline GeSn Well \\
\hline SiGeSn Barrier \\
\hline SiGeSn SCH \\
\hline GeSn Buffer \\
\hline Si substrate \\
\hline
\end{tabular}

Fig. 1. Layer structure.

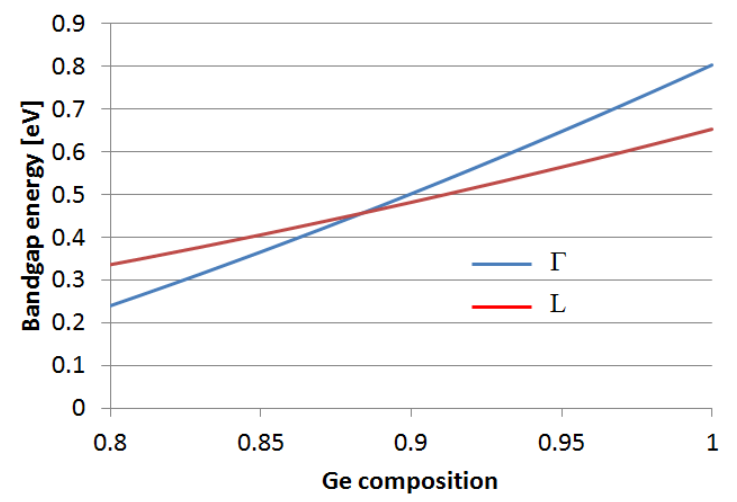

(a)

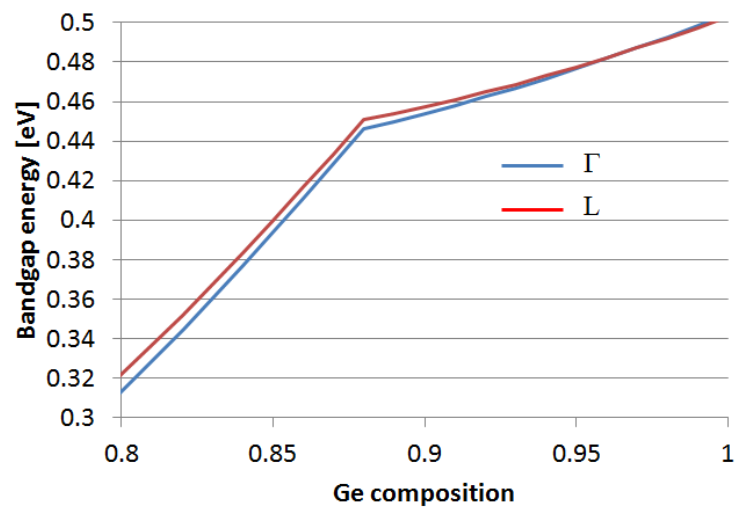

(b)

Fig. 2. Bulk bandgap energy of (a) GeSn and (b) GeSn on $\mathrm{Ge}_{0.88} \mathrm{Sn}_{0.12}$ buffer as a function of Ge composition.

band is assumed to be equal to the electron density and is given by

$$
N_{h}=\sum_{l} \int \frac{k_{t}}{\pi L_{w}} f_{h}\left(E_{\Gamma, l}\right) d k_{t}
$$

where $l$ is the QW subband number of valence band and $f_{h}(E)$ is the Fermi distribution for holes.

\section{B. Material gain}

In FCT, the material gain of QWs are calculated by Fermi's golden rule as

$$
g(\hbar \omega)=\frac{e^{2} \pi}{n_{B} C \varepsilon_{0} m_{0}^{2} \omega} \int d k_{t} \rho\left(k_{t}\right)|M|^{2}\left(f_{e}+f_{h}-1\right) L\left(E\left(k_{t}\right)-\hbar \omega\right)
$$

where $e$ is the electron charge, $\hbar$ is Plank's constant divided by $2 \pi, \varepsilon_{0}$ is the permittivity in vacuum, $n_{B}$ is the background refractive index, $m_{0}$ is the electron mass in vacuum, $\omega$ is the 
angular frequency, $\rho$ is the density of states, $|M|$ is the momentum matrix element. The function $L$ in (4) is called broadening function and used to "broaden" the optical spectrum to match the experimental spectrum. For example, Lorentz broadening function is given by

$$
L\left(E\left(k_{t}\right)-\hbar \omega\right)=\frac{(\hbar / \tau)^{2}}{(\hbar / \tau)^{2}+\left(E\left(k_{t}\right)-\hbar \omega\right)^{2}}
$$

where $\tau$ is the relaxation time. The type of the function (Lorentz, sech, etc) and the value of the relaxation time are chosen to fit the experimental spectrum. Although FCT is very easy to use for obtaining the material gain, the calculated spectrum shape and the magnitude of the material gain strongly depend on the function type and the value of relaxation time. Furthermore, it has been demonstrated that even if reasonable fitting parameters are used, the spectral position and shape are not consistent with the experiment $[11,13]$.

Her, we use MBT to obtain the material gain of QWs to exclude artificial fitting parameters to grasp inherent optical properties of QW both qualitatively and quantitatively. In MBT, by formulating the problem on the basis of second quantization, the so-called semiconductor Bloch equation (SBE) can be derived as

$$
\frac{d p_{k_{t}}}{d t}=-i \omega_{k_{t}} p_{k_{t}}-i \Omega_{k_{t}}\left(f_{e}+f_{h}-1\right)+\left.\frac{\partial p_{k_{t}}}{\partial t}\right|_{c o l}
$$

where $p_{k t}$ is the microscopic polarization, $\hbar \omega_{k t}$ is the renormalized transition energy, and $\Omega_{k t}$ contains the interaction term between the light and the carrier. The final term in (6) is the scattering contribution. Brief descriptions and numerical treatment of SBE are summarized in Appendix._By solving (6), the microscopic polarization is calculated and a macroscopic polarization, $P$, is obtained by summing the microscopic polarization over all the states. The macroscopic polarization is related to material gain through Maxwell's equations as

$$
g(\hbar \omega)=\operatorname{Im}\left[\frac{P}{\varepsilon_{0} n_{B}^{2} E_{0}}\right]=\operatorname{Im}\left[\frac{2}{\varepsilon_{0} n_{B}^{2} E_{0} V} \sum_{k_{t}} \mu_{k_{t}}^{*} p_{k_{t}}\right]
$$

where $V$ is the volume, $\mu_{k t}$ is the dipole matrix element, and $E_{0}$ is the electric field of light. The theory enables us to account for various important physical phenomena, such as the absorption peak due to excitons, bandgap renormalization, and the collapse of the absorption peak due to Coulomb screening effects, which cannot be considered in FCT.

Since only compressive strain is treated in this paper, only TE mode gain is considered. Room temperature (298K) is assumed for all the results.

\section{OPTICAL PROPERTIES OF GESN/SIGESN QUANTUM WELLS}

\section{A. Band structure and material gain}

Figure 3 shows the bandgap wavelength between first $\Gamma$ conduction subband and first valence subband (C1V1 wavelength, $\lambda_{\mathrm{ClV} 1}$ ) of GeSn QW as a function of the well thickness and the strain. The minus sign for the strain means compressive strain. Each curved line corresponds to the structure with a constant C1V1 wavelength. $\lambda_{\mathrm{C} 1 \mathrm{~V} 1}$ of GeSn QWs on $\mathrm{Ge}_{0.88} \mathrm{Sn}_{0.12}$ buffer is around 2500 to $3400 \mathrm{~nm}$, and therefore,

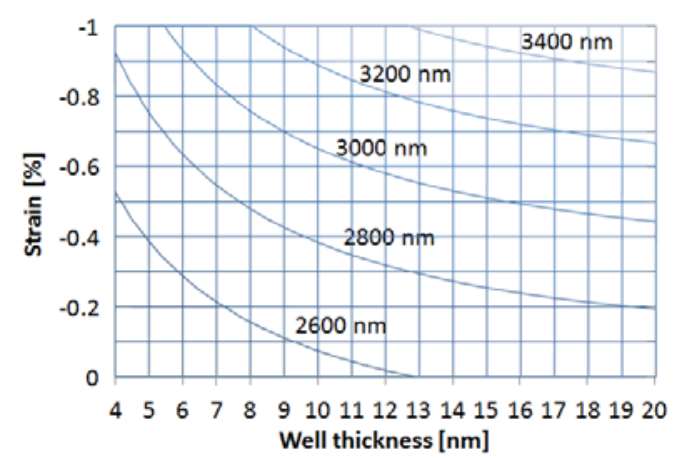

Fig. 3. C1V1 wavelength of GeSn QW as a function of the well thickness and the strain.

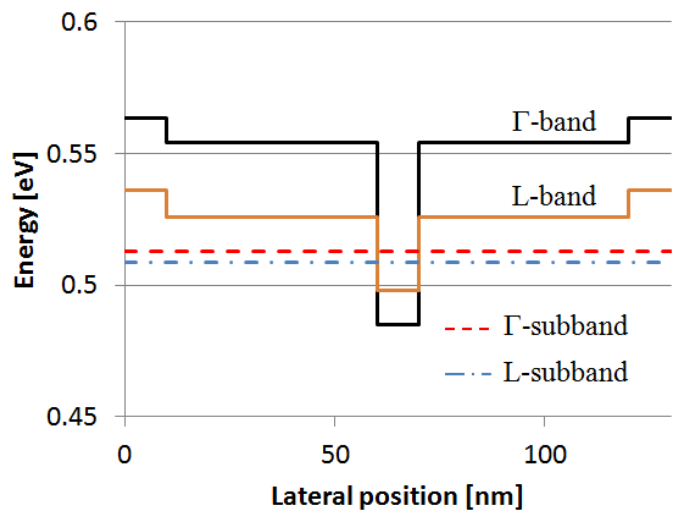

(a)

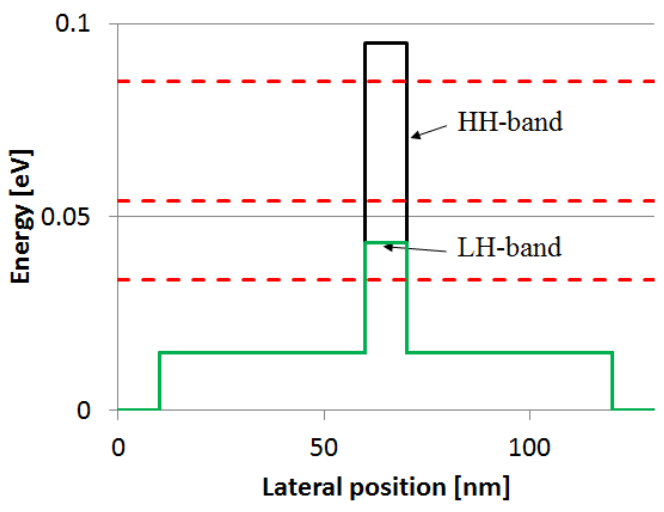

(b)

Fig. 4. (a) Conduction and (b) valence band potential profiles of GeSn QW with $L_{w}=10 \mathrm{~nm}$ and $\lambda_{\mathrm{C} 1 \mathrm{~V} 1}=2900 \mathrm{~nm}$.

GeSn QW is suitable for mid-IR light sources. Solid lines in Fig. 4(a) show the conduction band potential profile of GeSn QW with $L_{w}=10 \mathrm{~nm}$ and $\lambda_{\mathrm{C} 1 \mathrm{v} 1}=2900 \mathrm{~nm}$ of $\Gamma$ - and L-bands. Horizontal dashed and dash-dot lines are first conduction subband energy levels of $\Gamma$ - and L-bands. Although the well material is direct gap for bulk (L-bandedge is energetically larger than that of $\Gamma$-bandedge), for QW, the $\mathrm{L}$-subband energy is lower than that of $\Gamma$-subband, and hence, the $\mathrm{QW}$ is "quasi-direct" transition. We define the first subband energy difference as

$$
\Delta E=E_{\Gamma 1}-E_{L 1}
$$

to discriminate "direct" and "quasi-direct” QWs. $E_{\Gamma 1}$ and $E_{L 1}$ are the first conduction subband energies of $\Gamma$ - and L-bands. If $\Delta E$ 


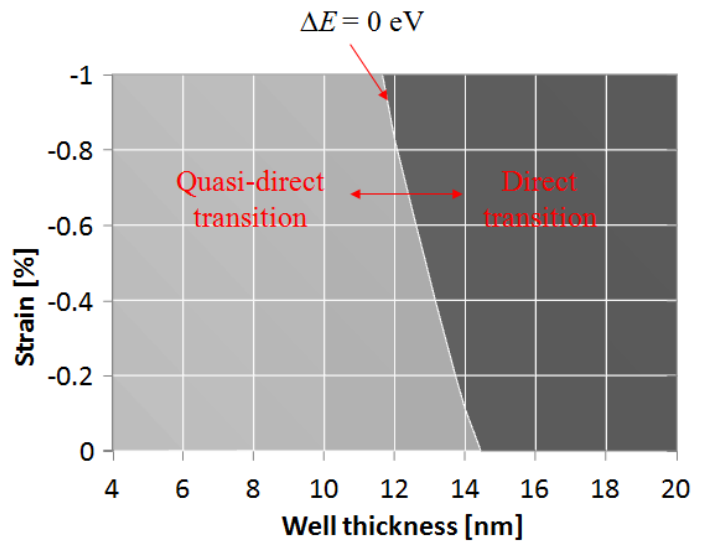

Fig. 5. $\Delta E$ as a function of the well thickness and the strain.

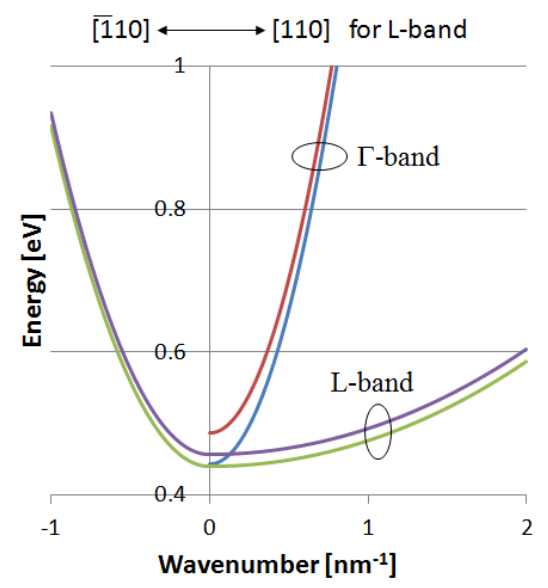

Fig. 6. Conduction band structure of GeSn QW with $L_{w}=10 \mathrm{~nm}$ and $\lambda_{\mathrm{C} 1 \mathrm{~V} 1}=2900 \mathrm{~nm}$.

is negative, the QW is truly direct gap. Figure 4 (b) shows the valence band (heaby- and light-hole : $\mathrm{HH}$ and $\mathrm{LH}$ ) profile of the same QW. The horizontal dased lines are quantum state of the well. Figure 5 shows $\Delta E$ as a function of the well thickness and the strain. The line for $\Delta E=0$ is plotted. Right hand side of the line is direct transition $(\Delta E<0)$ and the left hand side is quasi-direct transition $(\Delta E>0)$. Figure 6 shows the conduction $\Gamma$ - and L-band structures of GeSn QW shown in Fig. 4. Two band structures are plotted in the same graph for comparison. The effective mass of L-bandedge for [110] direction is very large, leading to the large density of states. Since the injected carriers are filled from lower energy side, carrier leakage to L-band is large for large $\Delta E$. Therefore, the small value of $\Delta E$ is preferable to increase the material gain. Figure 7 shows the carrier distribution in $\Gamma$ - and L-bands as a function of total carrier density. As expected, the most of carriers are distributed in L-band.

Figure 8(a) shows the material gain spectra of GeSn QW shown in Fig. 4 for $N_{e}=6 \times 10^{18} \mathrm{~cm}^{-3}$. Dashed lines are obtained by using FCT. Lorentz type broadening function is used and the spectra are calculated for three values of the relaxation times ( $\tau=0.05,0.1$, and $0.2 \mathrm{ps}$ ). As shown in the figure, the magnitude of material gain strongly depends on the value of the

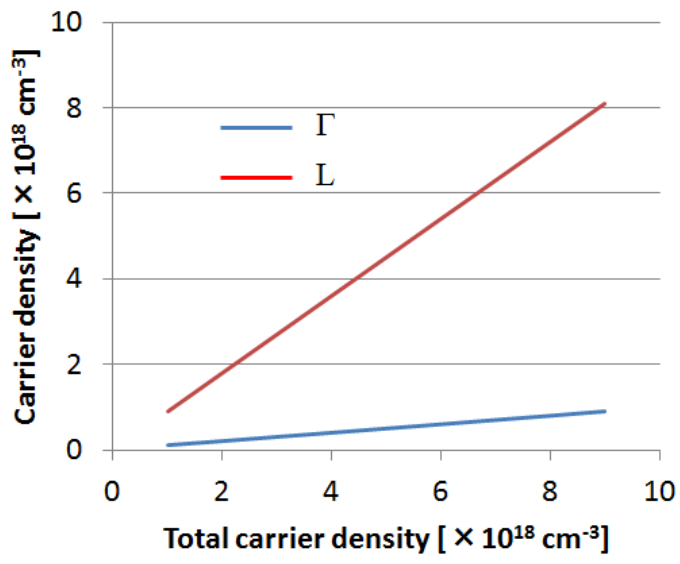

Fig. 7. Carrier density in $\Gamma$ - and L-bands as a function of total carrier density for GeSn QW with $L_{w}=10 \mathrm{~nm}$ and $\lambda_{\mathrm{C} 1 \mathrm{~V} 1}=2900 \mathrm{~nm}$.

relaxation time. The shape and the value are further changed if the different broadening function is used. Solid line in Fig. 8 (a) shows the material gain spectrum obtained by MBT. Without fitting parameters, smooth and unique gain spectrum is obtained. The spectral position is red shifted compared with those obtained by FCT. This is due to the bandgap wavelength correction originating from Coulomb scattering (bandgap renormalization) [13].

Since the band parameters of GeSn material (especially, bulk $\mathrm{Sn}$ ) are not fully understood, the effective mass dependence of the material gain spectra are investigated due to its direct effect on band structures, and hence, the value of material gain. Figure 8 (b) shows the material gain spectra of GeSn QW shown in Fig. 4 for $N_{e}=6 \times 10^{18} \mathrm{~cm}^{-3}$. For FCT, the relaxation time of $0.2 \mathrm{ps}$ is used because it gives the similar material gain value compared with MBT (see Fig. 8(a)). The effective mass of electron (both $\Gamma$ and $\mathrm{L}$ bands) and so-called Luttinger parameters [10] are changed by $\pm 10 \%$ and the change is denoted as $\Delta m$ in the Figure. Note that, for Luttinger parameters, if $\Delta m=+10 \%$, they are reduced $10 \%$ because the inverse of Luttinger parameters correspond to the effective mass of holes. From the Figure, if the effective mass is increased, the material gain spectra is red shifted and the value is reduced due to the increased density of states. For reduced effective mass, the inverse behavior can be seen. Even if the effective masses are changed $\pm 10 \%$, the difference between MBT and FCT can be seen in terms of spectral position. The change in the value of material gain is similar for both FCT and MBT. However, for FCT, the problem of how to choose the value of the relaxation time still remains.

Figure 8 (c) shows the material gain spectra for various carrier densities obtained by MBT. For lower carrier densities ( 3 and 5 $\times 10^{17} \mathrm{~cm}^{-3}$ ), there are exciton absorption peaks in the spectra, which is usually not taken into account in FCT. To include exciton absorption, for example, some non-variational approaches formulated in a momentum-space have been proposed $[18,19]$. However, they still need the relaxation time to broaden the exciton absorption peaks and cannot be used for large carrier density regime (gain regime). In MBT, as shown in 


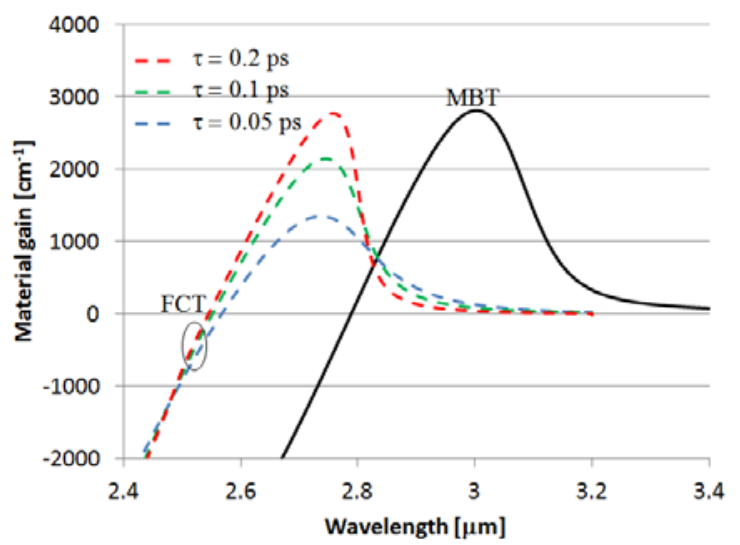

(a)

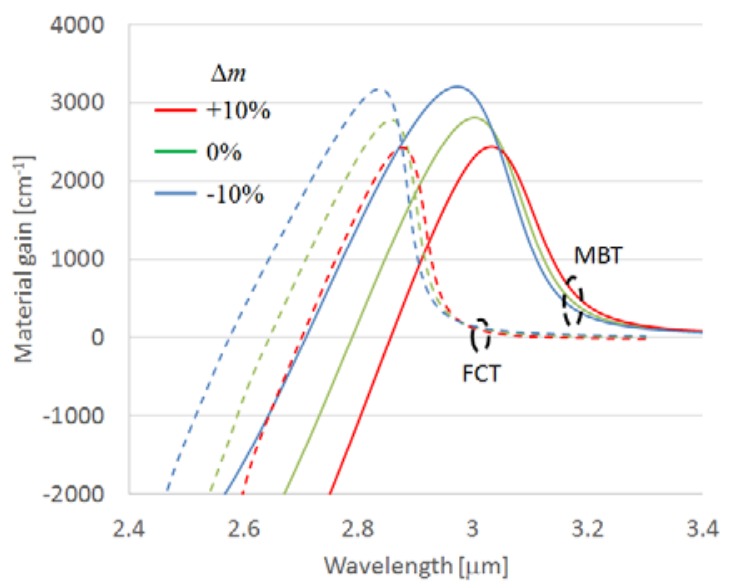

(b)

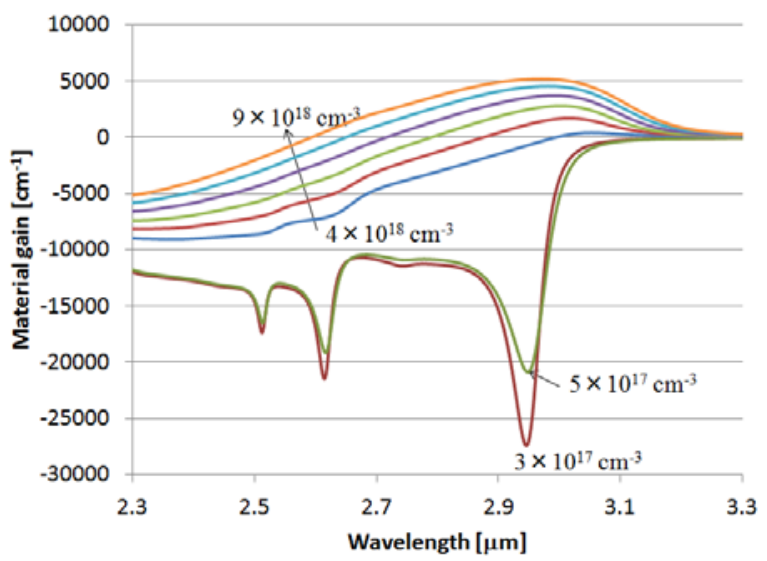

(b)

Fig. 8. (a) Gain spectra of GeSn QW with $L_{w}=10 \mathrm{~nm}$ and $\lambda_{\mathrm{C} 1 \mathrm{~V} 1}=$ $2900 \mathrm{~nm}$ at the carrier density of $6 \times 10^{18} \mathrm{~cm}^{-3}$. (b) Effective mass dependence of gain spectra of the same QW. (c) Gain spectra of the same QW for various carrier densities obtained by MBT. For larger carrier densities, gain spectra for 4 to $9 \times 10^{18} \mathrm{~cm}^{-3}$ are plotted with 1 $\times 10^{18} \mathrm{~cm}^{-3}$ interval.

Fig. 8, by increasing the carrier densities, the exciton peaks are collapsed due to Coulomb screening effects and the absorption turns into the gain for large carrier density. One of the advantages of MBT is that we can treat these phenomena simultaneously [10].

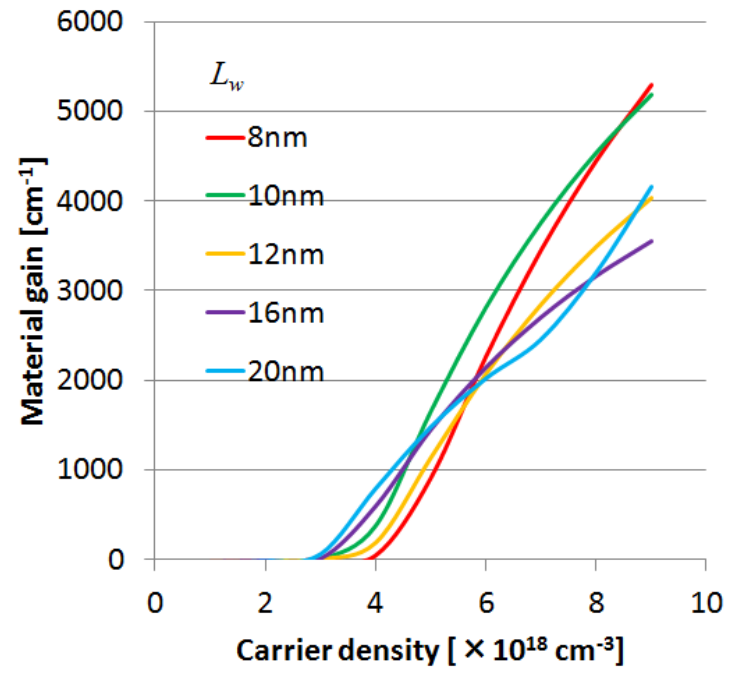

Fig. 9. Peak material gain as a function of carrier density for various values of $L_{w}$. For each structure, $\lambda_{\mathrm{C} 1 \mathrm{~V} 1}$ is fixed to $2900 \mathrm{~m}$.

\section{B. Structural dependence of material gain}

Figure 9 shows the peak material gain of GeSn QWs as a function of the carrier density for various values of $L_{w}$. $\lambda_{\mathrm{C} 1 \mathrm{~V} 1}$ is $2900 \mathrm{~nm}$ for all the structure (the strain is changed) and the results in this section are obtained by MBT. For large $L_{w}$, the transparent carrier density (at which the peak gain is zero) is small, however, the peak gain is small for large carrier density. This is due to the smaller density of states for large $L_{w}$.

For light source applications, the threshold carrier density, $N_{t h}$, and the differential gain at threshold, $d g /\left.d N\right|_{t h}$, are important parameters since $N_{t h}$ corresponds to the threshold current and the square root of the differential gain is proportional to the modulation speed. $N_{t h}$ is determined by

$$
\Gamma_{c} g_{t h}\left(N_{t h}\right)=\alpha_{i}+\alpha_{m}
$$

where $\alpha_{i}$ and $\alpha_{m}$ are internal and mirror losses, $g_{t h}$ is the threshold gain, and $\Gamma_{c}$ is the optical confinement factor of the waveguide. In [9], $g_{t h}$ was estimated to be $1100 \mathrm{~cm}^{-1}$ considering the mirror loss of the Fabry-Perot cavity and the absorption loss in doped layers for $\Gamma_{c}=7 \%$ waveguide. Since the loss in GeSn material system is not known well, $N_{t h}$ and $d g /\left.d N\right|_{t h}$, are investigated for $g_{t h}$ between 1000 to $2000 \mathrm{~cm}^{-1}$.

Figure 10 (a) shows $N_{t h}$ as a function of $L_{w}$ for $g_{t h}=1000$, 1500, and $2000 \mathrm{~cm}^{-1}$. Carrier distributions in L-band are neglected. $N_{t h}$ has its minimum around $L_{w}=10 \mathrm{~nm}$. For large $L_{w}$, since the saturated peak gain and the strain for the same $\lambda_{\mathrm{C} 1 \mathrm{~V} 1}$ are small (see Figs. 9 and 3), the material gain is small, leading to large $N_{t h}$. Figure 10 (b) shows $N_{t h}$ as a function of $L_{w}$ for $g_{t h}=$ 1000, 1500, and $2000 \mathrm{~cm}^{-1}$ with taking into account carrier distributions in L-band. Compared with Fig. 10 (a), for large $L_{w}$, the increase in $N_{t h}$ is suppressed and shows saturable behavior. This is because that for large $L_{w}, \Delta E$ is small and the QW becomes direct transition structure (see Fig. 5). Finally, Figure 10 (c) shows $d g /\left.d N\right|_{t h}$, as a function of $L_{w} \cdot d g /\left.d N\right|_{t h}$, has its maximum around $L_{w}=8$ to $10 \mathrm{~nm}$. Since the slope of peak gain curve in Fig. 9 is smaller for large $L_{w}$, the differential gain is also 
small. From Fig. 10, the well thickness of GeSn QWs should be about $10 \mathrm{~nm}$ to reduce $N_{t h}$ and increase $d g /\left.d N\right|_{t h}$ simultaneously.

It should be noted that although only one buffer layer $\left(\mathrm{Ge}_{0.88} \mathrm{Sn}_{0.12}\right)$ is considered in this paper, the same analysis can be done for other compositions. For larger values of Sn composition for buffer layer, the available wavelength is changed and the material gain may increase due to the direct gap nature of the material.

\section{CONCLUSION}

We have investigated the material gain of group IV $\mathrm{GeSn} / \mathrm{SiGeSn} \mathrm{QWs}$ for Si-photonics mid-IR light sources. MBT was used to obtain the material gain to exclude unknown fitting parameters included in conventional FCT. To treat Ge-based material, the carrier distribution in L-band is taken into account in MBT. The well thickness and the well strain dependence of the material gain was analyzed and the QW structure for obtaining large material gain is specified, namely, QW with large strain, and small $\Delta E$. It was shown that for the same C1V1 wavelength, the threshold carrier density is larger for small well thickness and shows saturable behavior for large well thickness due to the effect of $\Delta E$, which is not seen in conventional III-V QWs. From the calculated results, to obtain large material gain and differential gain simultaneously, the well thickness should be around $10 \mathrm{~nm}$.

\section{APPENDIX. BRIEF DESCRIPTION OF MBT AND NUMERICAL TREATMENT OF SBE}

Here, we briefly describe MBT and the numerical treatment of SBE. We start with Heisenberg's equation of motion

$$
i \hbar \frac{d O}{d t}=[H, O]
$$

where $O$ is the operator of physical quantity of interest and $H$ is the total Hamiltonian of the system. For treating many-body system, it is convenient to use second quantized Hamiltonian given by

$$
\begin{aligned}
& H=H_{F C T}+H_{C}+H_{L O} \\
& H_{F C T}=\sum_{\boldsymbol{k}}\left[\left(\varepsilon_{g 0}+\varepsilon_{e, \mathbf{k}}\right) a_{\boldsymbol{k}}^{\dagger} a_{\boldsymbol{k}}+\varepsilon_{h, \mathbf{k}} b_{-\boldsymbol{k}}^{\dagger} b_{-\boldsymbol{k}}\right. \\
& \left.-\left(\mu_{k} a_{k}^{\dagger} b_{-k}^{\dagger}+\mu_{k}^{*} b_{-k} a_{k}\right) E_{0}\right] \\
& H_{C}=\sum_{\boldsymbol{k}} \sum_{\boldsymbol{q} \neq 0} V_{s, q} b_{-\boldsymbol{k}}^{\dagger} b_{-\boldsymbol{k}}+\frac{1}{2} \sum_{\boldsymbol{k}, \boldsymbol{k}^{\prime}} \sum_{\boldsymbol{q} \neq 0} V_{s, q}\left(a_{\boldsymbol{k}+\boldsymbol{q}}^{\dagger} a_{\boldsymbol{k}^{\prime}-\boldsymbol{q}} a_{\boldsymbol{k}^{\prime}} a_{\boldsymbol{k}}\right. \\
& \left.+b_{\boldsymbol{k}+q}^{\dagger} b_{\boldsymbol{k}^{\prime}-q} b_{\boldsymbol{k}^{\prime}} b_{\boldsymbol{k}}-2 a_{\boldsymbol{k}+\boldsymbol{q}}^{\dagger} b_{\boldsymbol{k}^{\prime}-q}^{\dagger} b_{\boldsymbol{k}^{\prime}} a_{\boldsymbol{k}}\right) \\
& H_{L O}=\sum_{k} \sum_{\boldsymbol{q} \neq 0} \hbar g_{\boldsymbol{q}}\left[a_{\boldsymbol{k}+\boldsymbol{q}}^{\dagger} a_{\boldsymbol{k}}\left(c_{\boldsymbol{q}}+c_{\boldsymbol{q}}^{\dagger}\right)+b_{-\boldsymbol{k}}^{\dagger} b_{-\boldsymbol{k}-\boldsymbol{q}}\left(c_{\boldsymbol{q}}+c_{\boldsymbol{q}}^{\dagger}\right)\right]
\end{aligned}
$$

where $H_{F C T}, H_{C}$ and $H_{L O}$ are the Hamiltonian for free-carrier kinetics and interaction between free-carrier and light, Coulomb interaction, and longitudinal-optical (LO) phonon interaction (Frohlich Hamiltonian). $a_{k}, b_{k}$ and $a_{k}^{\dagger}, b_{k}^{\dagger}$ are annihilation and creation operators for electrons and holes. $\varepsilon_{g, 0}, \varepsilon_{e, k}$, and $\varepsilon_{h, k}$ are the bandgap of the material at $\Gamma$-point, electron energy, and hole energy, respectively. $\boldsymbol{q}=$ $\boldsymbol{k}$ '-k. $V_{s, q}$ is the screened Coulomb potential for QWs including form-factor contribution given by

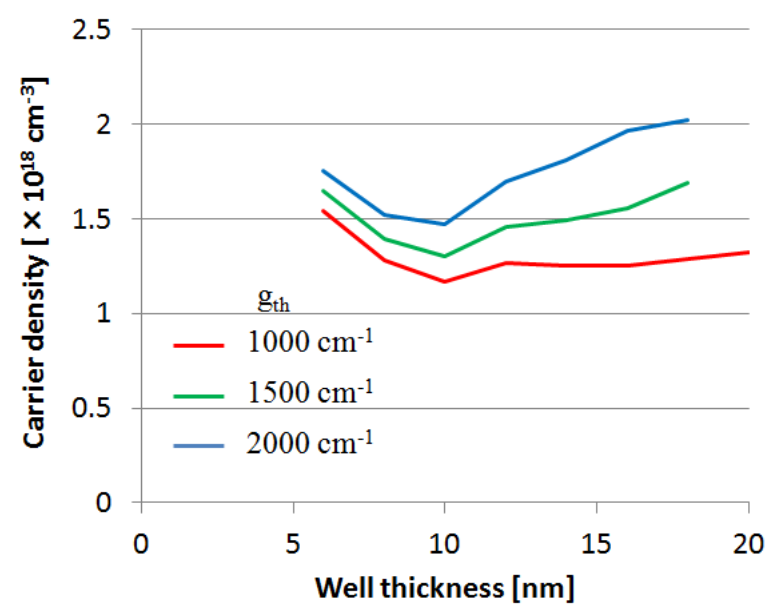

(a)

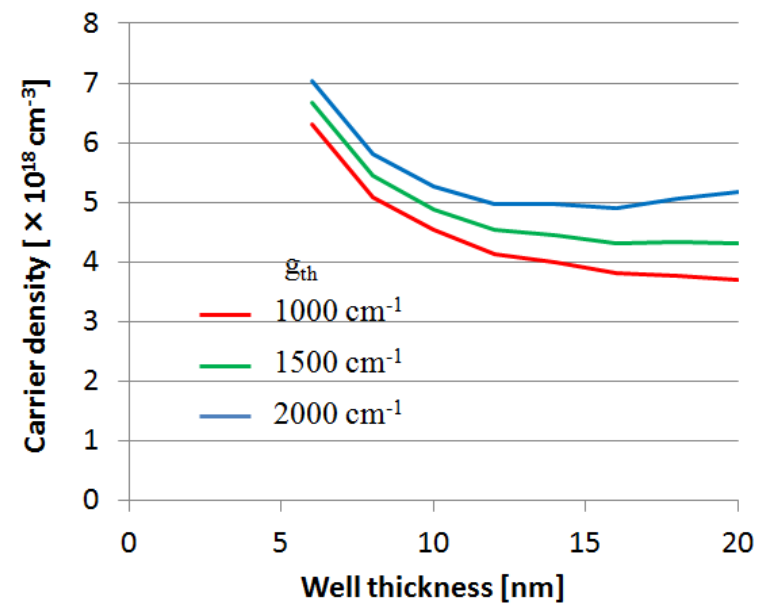

(b)

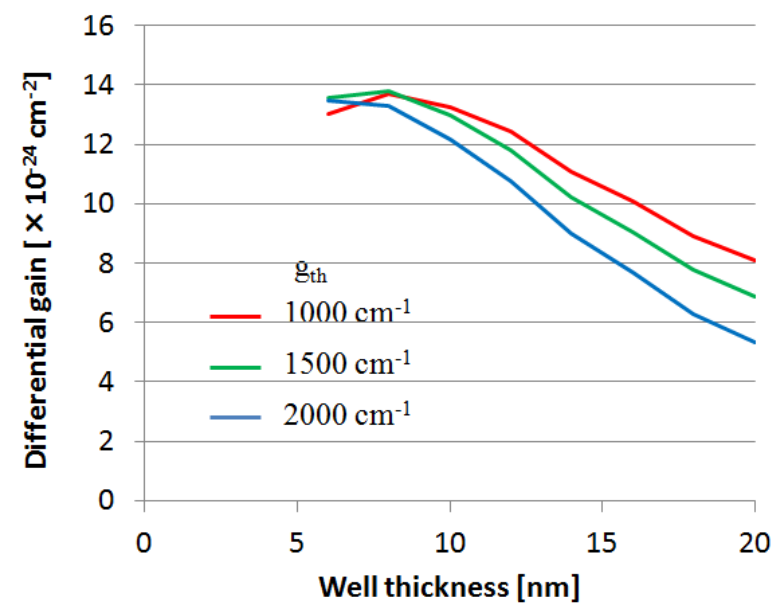

(c)

Fig. 10. $N_{t h}$ as a function of $L_{w}$ (a) without and (b) with the L-band contribution. (c) $d g /\left.d N\right|_{t h}$ as a function of $L_{\mathrm{w}}$.

$$
V_{s, q}=\frac{e^{2} f_{q}}{2 \varepsilon_{0} \varepsilon_{B} q A \varepsilon(q)}
$$

where $\varepsilon_{\mathrm{B}}$ is the background dielectric constant, $A$ is the area, $\varepsilon(q)$ is the dielectric function given by Lindhard formula, and $f_{q}$ is the form factor calculated by wavefunctions of QW. $c_{q}$ and $c_{q}^{\dagger}$ are the 
annihilation and creation operators of LO phonon. $g_{q}$ is the interaction matrix element given by

$$
\left|g_{\boldsymbol{q}}\right|^{2}=\frac{\omega_{L O} V_{s, q}}{2 \hbar}\left(\frac{1}{\varepsilon_{\infty}}-\frac{1}{\varepsilon_{s}}\right)
$$

where $\hbar \omega_{\mathrm{LO}}$ is the energy of LO phonon, $\varepsilon_{\infty}$ and $\varepsilon_{\mathrm{S}}$ are the high-frequency and static dielectric constants. Since we are interested in the material gain of QWs, $O$ is replaced with the operator for microscopic polarization, $b_{-k} a_{k}$. By evaluating the commutation of $H$ and $O$, we finally obtain SBE as (6). The full derivation of (6) is too lengthy to show here and the details can be found in, for example, [10] and [20]. Each term of (6) is given by

$$
\begin{aligned}
& p_{k_{t}}=\left\langle b_{-k_{t}} a_{k_{t}}\right\rangle \\
& \hbar \omega_{k_{t}}=\hbar \omega_{k_{t}}^{\prime}-\sum_{q} V_{s, q}\left(f_{e, k_{t}-q}+f_{h, k_{t}-q}\right) \\
& \Omega_{k_{t}}=\frac{1}{\hbar}\left(\mu_{k_{t}} E_{0}+\sum_{q} V_{s, q} p_{\boldsymbol{k}_{t}-\boldsymbol{q}}\right) \\
& \left.\frac{\partial p_{k_{t}}}{\partial t}\right|_{\text {col }}=\left.\frac{\partial p_{k_{t}}}{\partial t}\right|_{\text {Diag, } C}+\left.\frac{\partial p_{k_{t}}}{\partial t}\right|_{\text {nonDiag, } C}+\left.\frac{\partial p_{k_{t}}}{\partial t}\right|_{\text {Diag, LO }}+\left.\frac{\partial p_{k_{t}}}{\partial t}\right|_{\text {nonDiag, } L O}
\end{aligned}
$$

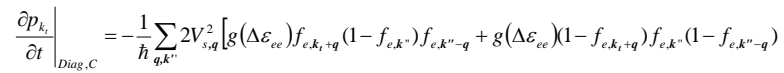

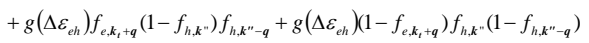

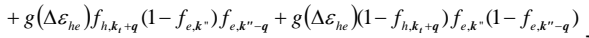

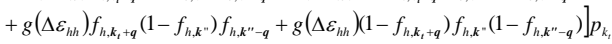

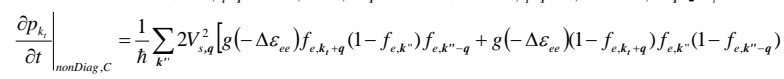

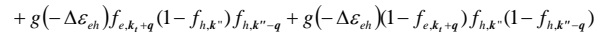

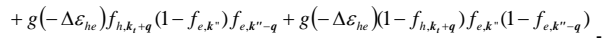

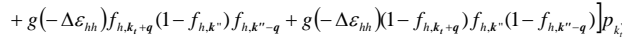

$$
\begin{aligned}
& \Delta \varepsilon_{a b}=\varepsilon_{a, \boldsymbol{k}_{t}}+\varepsilon_{b, \mathbf{k}^{\prime \prime}}-\varepsilon_{a, \boldsymbol{k}_{t}+\boldsymbol{q}}-\varepsilon_{b, \mathbf{k}^{\prime \prime}-q} \quad(a, b=e, h) \\
& \left.\frac{\partial p_{k_{k}}}{\partial t}\right|_{\text {Diag, LO }}=-\hbar \sum_{k^{\prime}} g_{q}^{2}\left\{g ( \Delta \varepsilon _ { e k _ { t } } ^ { + } ) \left[N_{q}\left(1-f_{e, k_{t}-q}\right)+\left(N_{q}+1\right) f_{e, k_{t}-q}\right.\right. \\
& +g\left(\Delta \varepsilon_{e k_{t}}^{-}\right)\left[\left(N_{q}+1\right)\left(1-f_{e, k_{t}-q}\right)+N_{q} f_{e, k_{i}-q}\right. \\
& +g\left(\Delta \varepsilon_{h k_{t}-q}^{+}\right)\left[N_{q}\left(1-f_{h, k_{t}-q}\right)+\left(N_{q}+1\right) f_{h, k_{t}-q}\right. \\
& +g\left(\Delta \varepsilon_{h k_{t}-q}^{-}\right)\left[\left(N_{q}+1\right)\left(1-f_{h, k_{-}-q}\right)+N_{q} f_{h, k_{i}-q}\right\} p_{k_{i}} \\
& \left.\frac{\partial p_{k_{t}}}{\partial t}\right|_{\text {nonDiag, LO }}=\hbar \sum_{k^{\prime}} g_{q}^{2}\left\{g ( \Delta \varepsilon _ { e k _ { k ^ { \prime } } - q } ^ { + } ) \left[N_{q}\left(1-f_{e, k_{t}}\right)+\left(N_{q}+1\right) f_{e, k_{t}}\right.\right. \\
& +g\left(\Delta \varepsilon_{e k_{t}-q}^{-}\right)\left(\left(N_{q}+1\right)\left(1-f_{e, k_{t}}\right)+N_{q} f_{e, k_{t}}\right. \\
& +g\left(\Delta \varepsilon_{h k_{i}}^{+}\right)\left[N_{q}\left(1-f_{h, k_{i}}\right)+\left(N_{q}+1\right) f_{h, k_{i}}\right. \\
& +g\left(\Delta \varepsilon_{h k_{t}}^{-}\right)\left[\left(N_{q}+1\right)\left(1-f_{h, k_{t}}\right)+N_{q} f_{h, k_{t}}\right\} p_{k_{i}} \\
& \Delta \varepsilon_{a k_{t}}^{ \pm}=\varepsilon_{a, k_{t}}-\varepsilon_{a, \boldsymbol{k}_{t}-\boldsymbol{q}} \pm \hbar \omega_{L O} \quad(a, b=e, h) \\
& \Delta \varepsilon_{a k_{t}-\boldsymbol{q}}^{ \pm}=\varepsilon_{a, \boldsymbol{k}_{t}-\boldsymbol{q}}-\varepsilon_{a, \boldsymbol{k}_{t}} \pm \hbar \omega_{L O} \quad(a, b=e, h)
\end{aligned}
$$

where $g(\Delta \varepsilon)$ is the generalized Heitler-zeta function [20-22], and $N_{q}$ is the Bose function describing the distribution of LO phonons. $\hbar \omega_{k t}{ }^{\prime}=\varepsilon_{g, 0}{ }^{+} \varepsilon_{e, k t}{ }^{+} \varepsilon_{h, k t}$. The correlation term (A11) can be divided into diagonal and nondiagonal components for given $k_{t}$ as $(\mathrm{A} 12,13,15,16)$.

Once we obtain (6), the microscopic polarization is obtained by solving (6). Since we are interested in steady-state gain, the left-hand-side of (6) is zero. The summations included in (A9) to (A16) are replaced with the integral with respect to the wavenumber as,

$$
\sum_{k^{\prime}} \rightarrow \frac{4 \pi^{2}}{A} \int_{0}^{\infty} d k^{\prime} \int_{0}^{2 \pi} d \phi
$$

By discretizing in k-space, each term can be evaluated numerically for given $k_{t}$ by using, for example, a trapezoidal rule for the integration. Again, more details of numerical treatment of these terms can be found in [10]. These procedure leads to a simultaneous equation for discretized $p_{k t}$ and by solving the equation, we obtain the microscopic polarization. Finally, the material gain is obtained by (7).

\section{REFERENCES}

[1] R. Soref, "Mid-infrared photonics in silicon and germanium," Nature Photon., vol.4, pp.495-497, Aug. 2010.

[2] B. Corbett, M. R. Gleeson, N. Ye, C. Robert, H. Yang, H. Zhang, N. Mac Suibhne, P. E.Morrissey, K. Thomas, A. Gocalinska, E. Pelucchi, R. Phelan, B. Kelly, J. O’Carroll, F. H. Peters and F.C. Garcia Gunning, "InP based active and passive components for communication systems at 2 $\mu \mathrm{m}$," in Proc. ECOC2014, Tu.4.4.5, 2014.

[3] G. Roelkens, U.D. Dave, A. Gassenq, N. Hattasan, C. Hu, B. Kuyken, F. Leo, A. Malik, M. Muneeb, E. Ryckeboer, D. Sanchez, S. Uvin, R. Wang, Z. Hens, R. Baets, Y. Shimura, F. Gencarelli, B. Vincent, R. Loo, J.V. Campenhout, L. Cerutti, J.-B. Rodriguez, E. Tourni'e, X. Chen, M. Nedeljkovic, G. Mashanovich, Li Shen, N. Healy, A.C. Peacock, X. Liu, R. Osgood, and William M. J. Green, "Silicon-based photonic integration beyond the telecommunication wavelength range," IEEE J. Sel. Top. Quantum Electron., vol. 20, pp.8201511, July 2014.

[4] R. E. Camacho-Aguilera,Y. Cai, N. Patel, J. T. Bessette, M. Romagnoli, L.C. Kimerling, and J. Michel, "An electrically pumped germanium laser,” Opt. Exp., vol. 20, pp. 11316-11320, May 2012.

[5] J. Mathews, R. T. Beeler, J. Tolle, C. Xu, R. Roucka, J. Kouvetakis, and J. Menéndez, "Direct-gap photoluminescence with tunable emission wavelength in $\mathrm{Ge}_{1-\mathrm{y}} \mathrm{Sn}_{\mathrm{y}}$ alloys on silicon," Appl. Phys. Lett., vol. 97, pp. 221912, Nov. 2010.

[6] G. Grzybowski, R. T. Beeler, L. Jiang, D. J. Smith, J. Kouvetakis, and J. Menéndez, "Next generation of $\mathrm{Ge}_{1-\mathrm{y}} \mathrm{Sn}_{\mathrm{y}}(\mathrm{y}=0.01-0.09)$ alloys grown on $\mathrm{Si}(100)$ via $\mathrm{Ge}_{3} \mathrm{H}_{8}$ and $\mathrm{SnD}_{4}$ : Reaction kinetics and tunable emission", Appl. Phys. Lett., vol. 101, pp. 072105, Aug. 2012.

[7] W. Kobayashi, T. Fujisawa, K. Tsuzuki, Y. Ohiso, T. Itoh, S. Kanazawa, T. Yamanaka, and H. Sanjoh, "Design and fabrication of wide wavelength range 25.8-Gb/s, 1.3- $\mu \mathrm{m}$, push-pull-driven DMLs,” IEEE/OSA Journal of Lightwave Technology, vol.32, pp.3-9, Jan. 2014.

[8] Y.-H. Zhu, Q. Xu, W.-J. Fan, and J.-W. Wang, "Theoretical gain of strained $\mathrm{GeSn}_{0.02} / \mathrm{Ge}_{1-\mathrm{x}-\mathrm{y}} \mathrm{Si}_{\mathrm{x}} \mathrm{Sn}_{\mathrm{y}}$ ' quantum well laser,” J. Appl. Phys., vol. 107, pp. 073108, 2010.

[9] G.-E. Chang, S.-W. Chang, and S.L. Chuang, "Strain-balanced $\mathrm{Ge}_{\mathrm{z}} \mathrm{Sn}_{1-\mathrm{z}}-\mathrm{Si}_{\mathrm{x}} \mathrm{Ge}_{\mathrm{y}} \mathrm{Sn}_{1-\mathrm{x}-\mathrm{y}}$ multiple-quantum-well lasers," IEEE J. Quantum Electron., vol. 46, pp.1813-1820, Dec. 2010.

[10] W.W. Chow and S.W. Koch, "Semiconductor-laser fundamentals," Springer, 1993.

[11] W.W. Chow, A. Knorr, S. Hughes, A. Girndt, and S.W. Koch, "Carrier correlation effects in a quantum-well semiconductor laser medium," IEEE J. Sel. Top. Quantum Electron., vol. 3, pp.136-141, Apr. 1997.

[12] T. Fujisawa, M. Arai, T. Yamanaka, Y. Kondo, and F. Kano, "Microscopic design of GaInNAs quantum well laser diodes on ternary substrates for high-speed and high-temperature operations,” J. Appl. Phys., vol. 105, pp. 113114, June 2009.

[13] T. Fujisawa, T. Sato, M. Mitsuhara, T. Kakitsuka, T. Yamanaka, Y. Kondo, and F. Kano, "Successful application of the 8-band $\boldsymbol{k} \cdot \boldsymbol{p}$ theory to optical properties of highly strained $\mathrm{In}(\mathrm{Ga}) \mathrm{As} / \mathrm{InGaAs}$ quantum wells with strong conduction-valence band coupling," IEEE J. Quantum Electron., vol. 45, pp.1183-1191, Sep. 2009.

[14] T. Fujisawa, M. Arai, and F. Kano, "Many-body design of highly strained GaInNAs electroabsorption modulators on GaInAs ternary substrates," $J$. Appl. Phys., vol. 107, pp. 093107, May 2010. 
[15] T. Fujisawa, T. Yamanaka, T. Tadokoro, N. Fujiwara, M. Arai, W. Kobayashi, Y. Kawaguchi, K. Tsuzuki, and F. Kano, "Theoretical and experimental investigations of the incident-power-dependent extinction ratio of electroabsorption modulator integrated with DFB laser," IEEE J. Quantum Electron., vol. 47, pp.60-65, Jan. 2011.

[16] S.-W. Chang, and S.L. Chuang, "Theory of optical gain of Ge-Si $\mathrm{Ge}_{\mathrm{y}} \mathrm{Sn}_{1-\mathrm{x}-\mathrm{y}}$ quantum-well lasers," IEEE J. Quantum Electron., vol. 43, pp.249-256, Mar. 2007.

[17] C. G. V. de Walle, "Band lineups and deformation potentials in the model-solid theory,” Phys. Rev. B, vol. 39, pp. 1871-1883, Jan.1989.

[18] J.P. Loehr and J. Singh, "Nonvariational numerical calculations of excitonic properties in quantum wells in the presence of strain, electric fields, and free carriers,” Phys. Rev. B, vol. 42, pp. 7154-7162, Oct. 1990.

[19] C.Y.-P. Chao and S.L. Chang, "Momentum-space solution of exciton states and heavy-hole-light-hole mixing in quantum wells," Phys. Rev. B, vol. 48, pp. 8210-8221, Sep. 1993.

[20] H. Haug and A.P. Jauho, "Quantum kinetics in transport and optics of semiconductors,” Springer, 2008.

[21] A. Girndt, F. Jahnke, A. Knorr, S.W. Koch, and W.W. Chow, "Multi-band Bloch equations and gain spectra of highly excited II-VI semiconductor quantum wells," Physica Stat. Sol. B, vol. 202, pp.725-739, Aug. 1997.

[22] J. Hader, S.W. Koch, and J.V. Moloney, "Microscopic theory of gain and spontaneous emission in GaInNAs laser material," Solid State Electron., vol. 47, pp.513-521, Mar. 2003.

Takeshi Fujisawa (M’05) was born in Sapporo, Japan, on January 12, 1979.He received the B.E., M.E., and Ph.D. degrees in electronic engineering from Hokkaido University, Sapporo, in 2001, 2003, and 2005, respectively. He was a Research Fellow of the Japan Society for the Promotion of Science from 2003 to 2006. In 2006, he joined the Nippon Telegraph and Telephone (NTT) Photonics Laboratories, NTT Corporation, Atsugi, Japan. In 2014, he became an Associate Professor at the Graduate School of Information and Science Technology, Hokkaido University. His research interests include active optical devices for optical communication systems, such as semiconductor lasers and modulators, and theoretical modeling of optical fibers and devices. He is an author or coauthor of more than 50 papers in international refereed journals.

Prof. Fujisawa has been served as an Associate Editor of Optics Express from 2014 and is a Member of the Institute of Electronics, Information and Communication Engineers of Japan.

Kunimasa Saitoh (S’00-M’01) received the B.S., M.S., and Ph.D. degrees in electronic engineering from Hokkaido University, Sapporo, Japan, in 1997, 1999, and 2001, respectively. From 1999 to 2001, he was a Research Fellow of the Japan Society for the Promotion of Science. From 2001 to 2005, he was a Research Associate with the Graduate School of Engineering, Hokkaido University. From 2005 to 2013, he was an Associate Professor at Graduate School of Information Science and Technology, Hokkaido University, and in 2013, he became a Professor there. He has been involved in research on fiber optics, nano-photonics, integrated optical devices, and computer-aided design and modeling of guided-wave devices using finite element method, beam propagation method, and so on. He is an author or coauthor of more than 150 research papers in refereed international journals. Prof. Saitoh is a Member of the Optical Society of America, and the Institute of Electronics, Information and Communication Engineers. He received the Excellent Paper Award and the Young Scientist Award from the IEICE, in 1999 and 2002, respectively, the Young Scientists' Prize of the Commendation for Science and Technology from the Ministry of Education, Culture, Sports, Science, and Technology (MEXT), Government of Japan in 2008. From 2009 to 2010, he served as a Secretary/Treasurer of the IEEE Sapporo Section. 